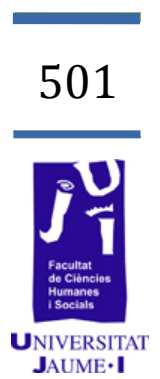

\title{
Análisis de una práctica educativa: hacia una escuela intercultural e inclusiva
}

Aida Sanahuja Ribés asanahuj@uji.es 
En este trabajo se reflexiona en torno a los elementos que caracterizan a la escuela intercultural e inclusiva, la cual consiste en hacer efectivo el derecho a la no discriminación y a la participación de todos los estudiantes, independientemente de su condición social o cultural, de su género o de sus características personales (Sales, Ferrández y Moliner, 2012). Se trata de un estudio de caso y se utiliza la observación participante como técnica de recogida de datos. Se describe de manera pormenorizada una práctica educativa, que fue llevada a cabo en un aula de educación infantil de 4 años del CEIP Isabel Ferrer de Castellón. En ella participaron 25 alumnos (10 chicas y 15 chicos), la maestra y cinco voluntarios. Se comentan aspectos relativos a la distribución del aula, a la estructura de las sesiones, a las técnicas de aprendizaje cooperativo utilizadas, a la especial atención prestada al lenguaje escrito y a la lectura y a la evaluación. Posteriormente se analiza la práctica educativa presentada siguiendo la dimensión $C$ de la guía CEIN para la construcción de la escuela intercultural inclusiva (Sales, Moliner y Traver, 2010). Se finaliza con las conclusiones, donde se remarca la importancia de avanzar hacia una escuela intercultural e inclusiva y se comentan las limitaciones del estudio.

Palabras clave: escuela intercultural e inclusiva, práctica educativa, relación escuela-comunidad, voluntariado, aprendizaje cooperativo.

\section{Introducción}

Es ampliamente compartida y aceptada la idea de que existe una gran diversidad $y$ heterogeneidad en las aulas. Tal y como introduce López Melero (2004) todos somos diferentes, no existen ni han existido dos seres humanos idénticos. Según Gómez Montes (2005), esta diversidad se puede resumir en torno a dos factores esenciales: 1) Factores de ámbito sociológico, derivados del propio contexto sociocultural y económico y originados por aspectos de carácter geográfico, étnico y religioso, y 2) Factores de ámbito psicopedagógico, derivados de las diferencias en las capacidades intelectuales de cada alumno, de la motivación por el aprendizaje, los intereses académicos y profesionales, los diferentes estilos de enseñanza y aprendizaje, etc. No obstante, en la práctica educativa de algunos docentes esta idea de diversidad parece ser ignorada. Esta concepción la reflejan muy bien Gregory y Chapman (2007) al introducir la analogía de que "una misma talla no sirve para todos», esto es, no podemos enseñar a todos los alumnos de la misma manera. O lo que es lo mismo en palabras de Beaudoin (2013), "la mayoría de las escuelas hacen que sus alumnos marchen al compás del mismo tambor, y el enfoque es diferente hay que tocar al compás de cada alumno». Esto no significa realizar una educación 
individualizada y descontextualizada de lo que realiza el grupo clase o recibir apoyos fuera del aula ordinaria. Aunque esta suele ser la respuesta educativa más común que se da al alumnado con discapacidad o dificultades de aprendizaje (Arnáiz, 2004). Más bien, se trata de entender y atender a la diversidad desde un marco en el que partiendo de la realidad seamos capaces de aplicar estrategias reales y perfectamente viables en nuestras aulas (Gómez Montes, 2005). Esto es, se deben introducir metodologías que propicien en el aula diferentes caminos de aprendizaje, acorde con las necesidades, intereses y estilos de aprendizaje de todo el alumnado, para ellos hay que entender la diversidad del aula como una dimensión que mejora y enriquece la práctica educativa (Moriña, 2008). Este propósito es el que persigue el modelo intercultural e inclusivo, «el cual consiste en hacer efectivo el derecho a la no discriminación y a la participación de todos los estudiantes, independientemente de su condición social o cultural, de su género o de sus características personales». (Sales, Ferrández y Moliner, 2012: 154)

Con el objetivo de avanzar hacia una escuela intercultural inclusiva surge la guía CEIN (Sales, Moliner y Traver, 2010) como un instrumento de autoevaluación escolar, que consta de tres dimensiones y de diversos factores clave. Cada factor introduce una serie de preguntas de reflexión para hacer una primera exploración de la realidad, en una segunda fase se toman decisiones colectivas sobre las dimensiones y factores seleccionados. En la Tabla 1, que se presenta a continuación, se recogen las tres dimensiones de dicha guía con sus respectivos factores.

Tabla 1. Dimensiones para la construcción de la Escuela Intercultural Inclusiva

(Sales, Moliner y Traver, 2010)

\section{A. Cómo somos (cómo nos vemos y cómo nos ven)}

A.1. Valores compartidos

A.2. Valoración positiva de la diversidad

A.3. Altas expectativas

B. Cómo nos organizamos

B.1. Proyecto educativo intercultural inclusivo

B.2. Liderazgo inclusivo

B.3. Cultura colaborativa

B.4. Tiempos y espacios para la reflexión y la innovación

B.5. Comunicación y mediación intercultural

B.6. Participación de la comunidad

C. Cómo aprendemos y enseñamos

C.1. Agrupamientos heterogéneos

C.2. Objetivos y contenidos curriculares por competencias

C.3. Metodología participativa y cooperativa

C.4. Actividades globalizadoras

C.5. Evaluación diversificada

C.6. Gestión dialógica del aula

C.7. Competencias de comunicación intercultural del profesorado

C.8. Desarrollo profesional y comunitario 
Una vez realizada esta breve contextualización teórica se pasará a presentar la metodología recurrida en este estudio y se describirá de manera pormenorizada una práctica educativa, comentando aspectos relativos a la distribución del aula, a la estructura de las sesiones, a las técnicas de aprendizaje cooperativo utilizadas, a la especial atención prestada al lenguaje escrito y a la lectura y a la evaluación. Posteriormente se analizará la práctica educativa presentada siguiendo la dimensión C de la guía CEIN.

\section{Material y método}

Este estudio se enmarca dentro de la metodología cualitativa. Se trata de un estudio de caso y se utiliza la observación participante como técnica de recogida de datos. Según Walker (1983: 45):

el estudio de caso es el examen de un ejemplo en acción. El estudio de unos incidentes y hechos específicos y la recogida selectiva de información de carácter biográfico, de personalidad, intenciones y valores, permite al que lo realiza captar y reflejar los elementos de una situación que le dan significado.

El método más importante de la etnografía es el de la observación participante. Este método permite que el investigador adopte un rol dentro del grupo y posibilita la observación desde la menor distancia posible (Woods, 1987).

En el caso que nos ocupa la observación participante fue realizada por una de las voluntarias participantes en la experiencia que se describe en el siguiente apartado de este trabajo.

\section{Descripción de la práctica educativa}

Esta práctica educativa fue llevada a cabo durante el primer y el segundo trimestre del curso académico 2011-2012 en un aula de infantil (4 años) del CEIP Isabel Ferrer de Castellón. El objetivo principal era iniciar en la lectura y la escritura a los alumnos, así como la cooperación y la participación de la comunidad educativa en el aula. En ella participaron 25 alumnos (15 niños y 10 niñas), la maestra y cinco voluntarios (un padre, dos madres y dos estudiantes de la Universitat Jaume I).

Primeramente, la maestra se puso en contacto con los voluntarios. Hizo un primer llamamiento a las familias y también a estudiantes de magisterio y psicopedagogía de la Universitat Jaume I. Una vez se contó con los cinco voluntarios necesarios, se realizó una reunión inicial, en la cual la maestra explicó cómo se coordinarían. Por lo que respecta a la organización general de trabajo, es necesario destacar que cada semana, antes de las sesiones, la maestra enviaba por correo electrónico un cuadro-resumen de lo que haría cada voluntario en cada grupo. $\mathrm{Y}$ al 
finalizar cada proyecto se reunieron los cinco voluntarios y la maestra para intercambiar impresiones y aportar propuestas de mejora.

Los voluntarios asistían al aula los jueves a primera hora de la mañana, después de las llamadas "rutinas» de infantil: pasar lista, ver el tiempo, organizar el trabajo del día,... hasta aproximadamente la hora del patio. Durante el curso se trabajaron dos proyectos: el proyecto de las tortugas (1.o trimestre) y el proyecto del agua (2.o trimestre). Ambos fueron escogidos mediante asamblea por el alumnado.

A continuación se procederá a explicar de manera detallada cómo se llevó a cabo la práctica educativa, mostrando la distribución del aula, la estructura de las sesiones, las técnicas de aprendizaje cooperativo que se utilizaron, la especial atención que se prestó a la escritura y a la lectura y finalmente se presentan aspectos relacionados con la evaluación.

\subsection{Distribución del aula}

Los alumnos estaban agrupados en cinco grupos heterogéneos de cinco miembros cada uno. Los cinco voluntarios pasaban por cada grupo, de manera que al terminar la sesión los cinco habían interactuado con todos los grupos. La maestra iba observando y guiando a quien lo necesitaba. Cada grupo realizaba una actividad concreta y disponía de una persona adulta encargada de dinamizarla. Aunque las actividades de cada grupo eran diferentes, mantenían relación entre ellas. La temática general era la misma y había sido votada en una asamblea por los propios alumnos, quienes habían decidido qué es lo que querían aprender.

Es importante remarcar que el espacio físico del aula tiene que facilitar la interacción de los alumnos tanto en su grupo como con el resto de los grupos (Sánchez Palomino y Carrión, 2002). La clase estaba distribuida como espacio versátil, tal y como se puede observar en la Figura 1. Con esta distribución se favorecía y se potenciaba la comunicación tanto en el grupo de trabajo como entre los grupos diferentes. De esta manera, al terminar cada sesión el portavoz del grupo explicaba a toda la clase que es lo que había trabajado su grupo, sin necesidad de cambiar de espacio físico.

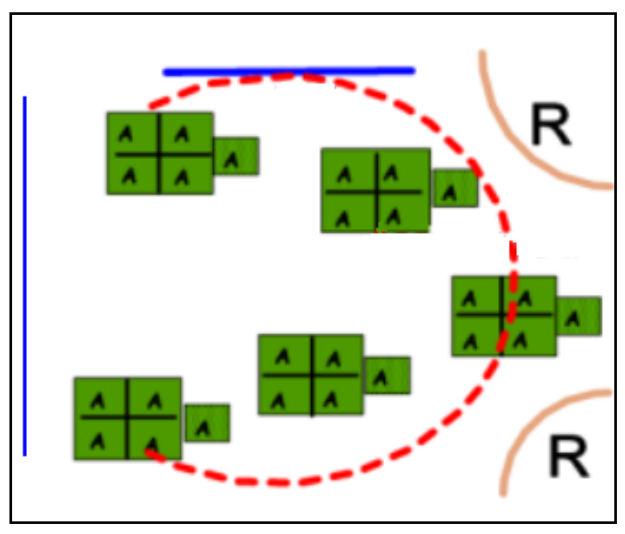

Figura 1. El aula como espacio versátil 


\subsection{Estructura de las sesiones}

En la Tabla 2, que se presenta a continuación, se recoge lo que hacía cada voluntario en cada subsesión. Cada sesión estaba dividida en cinco subsesiones y, como ya se ha dicho anteriormente, en cada subsesión los voluntarios estaban en un grupo diferente.

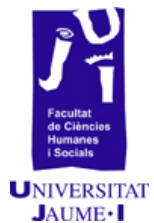

Tabla 2. Estructuración de las sesiones

\begin{tabular}{|c|c|c|}
\hline Subsesiones & Tarea a realizar & Tiempo estimado \\
\hline $\begin{array}{l}\text { Voluntario en la } \\
\text { subsesión } 1\end{array}$ & $\begin{array}{l}\text { Colaborar en el marcaje de los cargos. Y } \\
\text { supervisar el registro de estos por parte del } \\
\text { secretario del grupo. }\end{array}$ & Tiempo aproximado $5 \mathrm{~min}$. \\
\hline $\begin{array}{l}\text { Voluntario en la } \\
\text { subsesión } 2\end{array}$ & $\begin{array}{l}\text { Revisar con el secretario, en el plan del equipo, } \\
\text { los cargos. Remarcar que el plan del equipo } \\
\text { consiste en un cuaderno donde los equipos } \\
\text { hacen constar los cargos y funciones, las tareas } \\
\text { a realizar y las evaluaciones. } \\
\text { Ayudar en la definición del tema que van a } \\
\text { escribir. } \\
\text { Escribir, al dictado de las palabras, el texto } \\
\text { acordado entre todos. Animar a la escritura } \\
\text { individual en la hoja y supervisar que todos los } \\
\text { miembros del equipo colaboran e interactúan. }\end{array}$ & $\begin{array}{l}\text { Tiempo aproximado } 20 \\
\text { min. } \\
\text { Asegurarse que todos } \\
\text { tienen claro su cargo. } \\
\text { El responsable del } \\
\text { material trae el material } \\
\text { necesario. } \\
\text { ¿Sobre qué tema vamos a } \\
\text { escribir? ¿Qué } \\
\text { conocemos? } \\
\text { El voluntario escribe y los } \\
\text { alumnos copian. Ayudar } \\
\text { en la coherencia del texto. } \\
\text { Empieza a escribir el } \\
\text { portavoz y se hacen turnos } \\
\text { siguiendo las agujas del } \\
\text { reloj. Si se puede, } \\
\text { conviene que cada alumno } \\
\text { escriba una palabra } \\
\text { completa. } \\
\text { Tiempo aproximado } 5 \text { min. } \\
\text { El secretario saca el plan } \\
\text { del equipo y se van } \\
\text { registrando las respuestas } \\
\text { a las preguntas de } \\
\text { evaluación. Hacemos las } \\
\text { preguntas y esperamos la } \\
\text { reflexión y el diálogo del } \\
\text { equipo. Es necesario } \\
\text { asegurar la objetividad. }\end{array}$ \\
\hline
\end{tabular}




\begin{tabular}{|c|c|c|c|}
\hline $\begin{array}{l}\text { Voluntario en la } \\
\text { subsesión } 3\end{array}$ & $\begin{array}{l}\text { Preparar la exposición oral del contenido } \\
\text { trabajado y de la actividad que se ha realizado. }\end{array}$ & $\begin{array}{l}\text { Tiempo aproximado } 10 \\
\text { min } \\
\text { ¿Qué se ha } \\
\text { explicado/escrito en esta } \\
\text { hoja? ¿Se ha terminado el } \\
\text { trabajo o hay que } \\
\text { continuar? }\end{array}$ & \multirow[t]{4}{*}{ 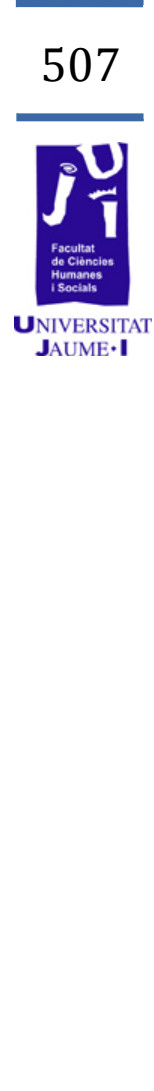 } \\
\hline $\begin{array}{l}\text { Voluntario en la } \\
\text { subsesión } 4\end{array}$ & $\begin{array}{l}\text { Escuchar al portavoz en la exposición ora } \\
\text { hacer preguntas. }\end{array}$ & \begin{tabular}{l|l} 
& Tiempo aproximado 5 min. \\
y & ¿Quién es el portavoz? \\
Explica que habéis hecho \\
en esta hoja.
\end{tabular} & \\
\hline \multicolumn{3}{|c|}{$\begin{array}{l}\text { Exposición oral del portavoz de cada equipo a todo el grupo clase } \\
2 \text { minutos por portavoz ( } 10 \mathrm{~min})\end{array}$} & \\
\hline $\begin{array}{l}\text { Voluntario en la } \\
\text { subsesión } 5\end{array}$ & $\begin{array}{l}\text { Ayudar en las reflexiones en referencia a la } \\
\text { expresión oral. }\end{array}$ & $\begin{array}{l}\text { Tiempo aproximado } 5 \text { min. } \\
\text { ¿El portavoz ha explicado } \\
\text { todo el trabajo? ¿Le ha } \\
\text { faltado explicar alguna cosa? } \\
\text { ¿Todos le hemos escuchado? } \\
\text { ¿Ha hablado en un tono de } \\
\text { voz adecuado y hemos podido } \\
\text { escucharle? ¿Le hemos } \\
\text { ayudado en la explicación? }\end{array}$ & \\
\hline
\end{tabular}

Esta estructura de trabajo se llevó a cabo en cada una de las sesiones, tanto en el proyecto de las tortugas como en el proyecto del agua.

\subsection{Aprendizaje cooperativo}

Según Pujolàs (2008), en un aula organizada en equipos cooperativos de trabajo, los alumnos aumentan su protagonismo y participan de una forma mucho más activa en el proceso de enseñanza y aprendizaje y en la gestión de la clase, y comparten con el profesorado la responsabilidad de enseñar, también ellos a sus propios compañeros. Los alumnos se convierten en individuos autónomos y se propicia un clima de aula muy favorable para el aprendizaje, puesto que se van dando las condiciones emocionales y relacionales imprescindibles para que los estudiantes puedan aprender efectivamente.

Seguidamente se abordaran las dos técnicas que se utilizaron en esta experiencia: la técnica de la instrucción compleja y el folio giratorio.

\subsubsection{Técnica de la Instrucción compleja}

La técnica de la instrucción compleja consiste en asignar a cada miembro del grupo un rol o responsabilidad con funciones claramente marcadas (Cohen, 1999). De esta manera, cada miembro del grupo se ocupa de la organización y la gestión del trabajo, de forma que se potencia la participación de todo el grupo. A medida que van avanzando 
las sesiones los roles van rotando. De esta manera cada miembro del grupo va desempeñando diferentes tareas cada vez.

Los cargos así como las tareas y responsabilidades de cada miembro del grupo se resumen en la Tabla 3, que se presenta a continuación.

Tabla 3. Cargos de los miembros del grupo

\begin{tabular}{|c|c|}
\hline Cargos & Tareas y responsabilidades \\
\hline PORTAVOZ & $\begin{array}{l}\text {-Anima a los miembros del equipo para avanzar en el trabajo. } \\
\text {-Habla en nombre de todo el equipo cuando se solicita la opinión } \\
\text { del grupo. } \\
\text {-Expone oralmente el trabajo realizado. } \\
\text {-Asume las funciones del secretario y del avisador cuando estos no } \\
\text { han venido. }\end{array}$ \\
\hline $\begin{array}{l}\text { ENCARGADO DE LA } \\
\text { LIMPIEZA }\end{array}$ & $\begin{array}{l}\text {-Mantiene el espacio de trabajo limpio y ordenado. } \\
\text {-Limpia las mesas después de trabajar. }\end{array}$ \\
\hline $\begin{array}{l}\text { RESPONSABLE DEL } \\
\text { MATERIAL }\end{array}$ & $\begin{array}{l}\text {-Proporciona los materiales necesarios. } \\
\text {-Cuida los materiales. } \\
\text {-Ordena y recoge los materiales. } \\
\text {-Asume las funciones del encargado de la limpieza cuando este no } \\
\text { ha venido. }\end{array}$ \\
\hline SECRETARIO & $\begin{array}{l}\text {-Rellena los formularios de registros de los cargos y de la } \\
\text { evaluación. } \\
\text {-Reparte las hojas. } \\
\text {-Asume las funciones del responsable del material y del portavoz } \\
\text { cuando estos no han venido. }\end{array}$ \\
\hline AVISADOR & $\begin{array}{l}\text {-Controla el tono de la voz de los miembros del equipo. } \\
\text {-Anuncia al portavoz si algún miembro del equipo no cumple con } \\
\text { sus responsabilidades. }\end{array}$ \\
\hline
\end{tabular}

\subsubsection{Folio giratorio}

El voluntario asigna una tarea al equipo (una lista de palabras relacionadas con el tema, las cosas que saben sobre ese tema, una frase que resuma una idea fundamental del tema estudiado,...) y un miembro del equipo empieza a escribir su parte o su aportación en un «folio giratorio». Mientras los demás se fijan en cómo lo hace, le ayudan si hace falta, le corrigen, le animan, etc. A continuación, pasa el folio al compañero que tiene al lado, siguiendo la dirección de las agujas del reloj para que escriba su parte de la tarea en el folio, y así sucesivamente hasta que todos los miembros del equipo han participado en la resolución de la tarea. 
Cada alumno puede escribir su parte con un rotulador de un determinado color (el mismo que ha utilizado para escribir su nombre en la parte de detrás del folio). Así a simple vista puede verse la aportación de cada uno (Pujolàs, 2008).

\subsection{Especial atención al lenguaje escrito y a la lectura}

Recordemos que uno de los principales objetivos del trabajo era la iniciación a la lectura y la escritura del alumnado de dicha clase. Así pues los voluntarios dedicaron en cada sesión especial atención a estos procesos. Las pautas fueron las siguientes:

- Escribir una hoja al dictado del texto de los niños.

- Leer lentamente cada palabra que se escribe y se les ayuda a:

- Dar coherencia al texto.

- Distinguir entre un texto oral (la explicación de una idea) y un texto escrito (cómo queremos escribir esa idea).

- Si la longitud del texto lo permite, se les ayuda a organizar los turnos para que cada alumno escriba una palabra entera. Si no, cada alumno escribe una letra de la palabra en cuestión.

- Empieza a escribir el secretario y, a continuación, se siguen los turnos según las agujas del reloj.

- Mientras uno escribe, los compañeros miran y ayudan, le corrigen, le dictan...

- Se revisa (leyendo) lo que se va escribiendo, para asegurarse de que se está escribiendo todo aquello que se quiere.

- Al terminar, se lee todo el texto.

\subsection{Evaluación}

Los voluntarios conjuntamente con la reflexión de los distintos miembros del grupo eran los encargados de realizar la evaluación de los alumnos, la cual quedaba recogida en el plan del equipo. En la Tabla 4, se agrupan algunos puntos que se tenían en cuenta para valorar cada apartado.

Tabla 4. Posibles ítems a tener en cuenta en la evaluación

\begin{tabular}{|l|l|}
\hline Apartado & \multicolumn{1}{|c|}{ Ítem a valorar } \\
\hline & -Conoce cuál es su cargo. \\
& -Se responsabiliza de sus funciones. \\
& -Conoce los cargos de los otros miembros del grupo. \\
1. Registro de los & -Reconoce los nombres de los compañeros para registrar los \\
cargos & $\begin{array}{l}\text { cargos correspondientes. } \\
\text {-Exige a sus compañeros que hagan uso de sus } \\
\end{array}$ \\
& responsabilidades. \\
\hline
\end{tabular}




\begin{tabular}{|c|c|}
\hline $\begin{array}{l}\text { 2. Escritura de la } \\
\text { información }\end{array}$ & $\begin{array}{l}\text {-Aporta ideas para escribir la información. } \\
\text {-Tiene iniciativa. } \\
\text {-Se implica en la tarea, colabora. } \\
\text {-Su escritura es lineal. } \\
\text { - Necesita mucha ayuda, poca o ninguna. } \\
\text {-Conoce el nombre de las letras para dictarlas a los compañeros. } \\
\text {-Hace hipótesis silábicas. } \\
\text {-... } \\
\text {-Es objetivo en la autoevaluación y coevaluación. } \\
\text { - Acepta las valoraciones de los otros compañeros. } \\
\text {-... }\end{array}$ \\
\hline $\begin{array}{l}\text { 3. Preparación de la } \\
\text { exposición oral }\end{array}$ & $\begin{array}{l}\text {-Participa en la explicación del trabajo realizado. } \\
\text {-Se centra en la información que se quiere escribir o en la } \\
\text { utilización del material, las ayudas, el proceso de escritura, pintar, } \\
\text { recortar,... } \\
\text {-... }\end{array}$ \\
\hline $\begin{array}{l}\text { 4. Preguntas al } \\
\text { portavoz para la } \\
\text { exposición oral }\end{array}$ & $\begin{array}{l}\text {-Contesta a las preguntas realizadas. } \\
\text {-Explica coherentemente la información. } \\
\text {-La explicación es correcta con el trabajo realizado. } \\
\text {-Los compañeros escuchan con atención la explicación del } \\
\text { portavoz. } \\
\text {-... }\end{array}$ \\
\hline $\begin{array}{l}\text { 5. Reflexiones sobre } \\
\text { la exposición oral } \\
\text { realizada }\end{array}$ & $\begin{array}{l}\text {-Lo ha explicado todo. } \\
\text {-El tono de voz era adecuado. } \\
\text {-Ha escuchado el portavoz mientras explicaba. } \\
\text {-Ha ayudado al portavoz en la explicación. } \\
\text {-... }\end{array}$ \\
\hline
\end{tabular}

Así pues al finalizar cada sesión, cada grupo conjuntamente con el voluntario correspondiente reflexionaba sobre cómo había trabajado el equipo de manera grupal e individual. Primeramente se reflexionaba conjuntamente sobre qué aspectos era necesario mejorar para el buen funcionamiento del grupo, por eso el secretario en el plan del equipo marcaba una cruz sobre lo que se había hecho (muy bien, bien o era necesario mejorar). Seguidamente, se pasaba a la evaluación individual, en este caso era el voluntario quien decidía objetivamente y teniendo en cuenta los criterios anteriormente presentados en la Tabla 4, quien pegaba el adhesivo (el cual tenía representado un grupo trabajando) en su casilla del plan del equipo, es decir, donde se indicaba su nombre, en función de:

- Su participación aportando ideas para escribir.

- La escucha de lo que comentaban los compañeros.

- La ayuda prestada a los compañeros en referencia a escribir palabras, recortar, enganchar, pintar...

- Si evitaba molestar en la mesa. Entendiendo la conducta de molestar como: el coger los materiales que están en la mesa 
cuando no los necesita nadie, hablar sobre otros temas no relacionados con el proyecto mientras un compañero trabaja sin ser ayudado y agobiar a los compañeros.

Se valoraba también la escucha de la exposición oral de los diferentes portavoces, de esta manera se enganchaba en el plan del equipo otra pegatina con una oreja dibujada. En cada sesión, cada niño podía obtener un máximo de dos adhesivos, uno que valoraba el trabajo realizado en la sesión y otro la escucha. Durante todo el proyecto los alumnos podían ver en el plan del equipo cómo trabajaban en grupo, cuántos adhesivos tenían de participación y cuántos en escucha a los compañeros.

\section{Análisis de la práctica educativa: hacia una escuela intercultural e inclusiva}

Tal y como se ha mencionado anteriormente, para analizar la práctica presentada se utilizará la guía CEIN para la construcción de la escuela intercultural inclusiva (Sales, Moliner y Traver, 2010). Al tratarse de una práctica educativa desarrollada en una sola aula de la escuela centraremos nuestro análisis en la dimensión C. Cómo aprendemos y enseñamos. A continuación, se procederá a comentar y desarrollar cada uno de los factores de la tercera dimensión de la guía.

C.1. Agrupamientos heterogéneos: a lo largo de la sesión se promueven principalmente dos tipos de agrupamientos: pequeño grupo y gran grupo/clase. El alumnado trabaja en un pequeño grupo dinamizado en cinco momentos diferentes por cinco voluntarios y al finalizar la sesión se promueve la participación en el gran grupo o grupo clase para que cada grupo pequeño comparta con el resto de la clase lo que han trabajado en esa sesión. De esta manera se promueven competencias para que el alumnado sepa trabajar en distintas modalidades de agrupamiento. Cabe destacar también que el criterio básico para crear los grupos de trabajo es la heterogeneidad, teniendo en cuenta el género, la lengua, las capacidades, los intereses, los ritmos... Además, cada alumno a lo largo de las diferentes sesiones va adoptando roles y funciones diferentes que deben desempeñar para el buen funcionamiento de este.

C.2. Objetivos y contenidos curriculares por competencias: los objetivos de aprendizaje son claros y se priorizan aquellos objetivos y contendidos más significativos y funcionales para el grupo-clase según los intereses y las curiosidades que el alumnado manifiesta en la asamblea.

C.3. Metodología participativa y cooperativa: con esta práctica educativa se fomenta tanto el aprendizaje intergrupal e intragrupal. Con los roles o cargos se da la oportunidad al alumnado de asumir diferentes responsabilidades y funciones que guían su propio aprendizaje. La principal metodología utilizada es la de los proyectos de trabajo. Se 
potencia que el alumnado explore puntos de vista diferentes al suyo, puesto que entre todos acuerdan lo que van a escribir. Como se ha mencionado en la descripción de la práctica, se fomenta el aprendizaje cooperativo y la maestra actúa como facilitadora del aprendizaje, destacando el papel dinamizador de los voluntarios, aprovechando así los recursos personales de la comunidad. Se utilizan diferentes fórmulas de motivación: primeramente cabe destacar que el proyecto nace de los propios intereses del alumnado que lo escoge mediante asamblea general, además en la evaluación se dan adhesivos para incentivar el trabajo cooperativo y la escucha a los compañeros. La presencia de voluntarios en el aula son por sí mismos otro elemento motivador para el alumnado. Así pues, a lo largo de las sesiones cada grupo va construyendo continuamente el aprendizaje de la temática que habían planteado y que quería aprender. El plan de trabajo permite al alumnado organizar y recoger los diferentes materiales e informaciones necesarias para la sesión.

C.4. Actividades globalizadoras: con esta práctica se potencia la globalización del aprendizaje a través de la participación en la búsqueda de los interrogantes previamente planteados por el propio alumnado. A lo largo de la sesión, el alumnado desempeña diferentes actividades que incluyen diferentes estrategias: organización del trabajo, desempeño de diferentes roles, discusión oral, escritura, dibujo, reflexión, exposición oral al gran grupo, etc. Durante la evaluación se proporciona feedback al alumnado sobre cómo ha trabajado tanto individual como grupalmente, sobre lo que ha aprendido y sobre lo que debería de realizar para mejorar.

C.5. Evaluación diversificada: en el momento de la evaluación se reflexiona sobre cómo se ha trabajado individual y grupalmente y se evalúa tanto en el proceso de aprendizaje como el producto. Se favorece la autoevaluación y la coevaluación, además se permite al voluntario participar en el proceso de evaluación. Este proceso propicia la igualdad evitando las clasificaciones y discriminaciones. Además de los conceptos, se evalúan los procedimientos y las actitudes. En la evaluación se propicia la reflexión, tanto individual como grupal, de cómo se ha trabajado, de los aspectos que han de mejorar, del trabajo pendiente, etc.

C.6. Gestión dialógica del aula: el voluntario provoca el intercambio y la comunicación entre todos los miembros del grupo. El ambiente, la organización y los recursos del aula contribuyen a la autonomía y responsabilidad del alumnado. Los problemas de conducta u organizacionales son comentados y reflexionados en la evaluación para ir fomentando poco a poco la mejora en proceso de enseñanza-aprendizaje. Los alumnos mediante la asamblea toman decisiones sobre su proceso de aprendizaje. 
C.7. Competencias de comunicación intercultural del profesorado: en esta práctica la maestra se encarga de facilitar y guiar a los grupos que lo necesitan, delegando todo el protagonismo en el alumnado y el voluntariado encargado de dinamizar cada uno de los grupos.

C.8. Desarrollo profesional y comunitario: a través de las reuniones entre la maestra y los voluntarios se adoptan las propuestas de mejora que se consideran, haciendo flexible la práctica educativa y reflexionando de manera colectiva sobre ella.

\section{Discusión y conclusiones}

La experiencia fue muy gratificante para todos los participantes (alumnos, maestra y voluntarios). Se consiguió una buena dinámica de trabajo y se observaron mejoras a medida que iban avanzando las sesiones tanto en el trabajo en grupo, como en la escucha y en los procesos de lectura y escritura. Cabe destacar que la eficacia de los grupos cooperativos se debe principalmente a la diversidad $y$ complementariedad de las funciones de todos los miembros (Pujolàs, 2003). Además, hay que subrayar de esta práctica educativa el hecho de permitir tanto a las familias como a la comunidad participar en ella. Tal y como introducen Stainback y Stainback (1999), para que se dé una verdadera inclusión es imprescindible la interrelación familia-escuelacomunidad. Así pues, mediante la práctica descrita se contribuyó a una mayor inclusión tanto social como educativa en el aula, ya que se logró la participación y la cooperación de todos. Los alumnos valoraban muy positivamente la presencia de personas adultas en el aula. $Y$ es que, tal como revelan otras investigaciones, la presencia de voluntarios en el aula acelera el aprendizaje del alumnado (Vieira Parra y Puigdellivol, 2013). Para los voluntarios esta experiencia también fue muy enriquecedora. Por una parte estaban los padres, quienes podían observar como aprendían sus hijos y como interactuaban con sus compañeros. Las estudiantes de la universidad pudieron contactar con la realidad del aula y conocer y valorar las implicaciones organizativas, metodológicas y de clima del aula desde el marco de la respuesta a la diversidad, de manera que esta experiencia tuvo una gran repercusión en su formación.

¡TODOS! Alumnos, maestra y voluntarios compartieron lo que sabían y aprendieron:

- Sobre el proyecto de las tortugas: qué beben y qué comen, cómo nacen, los reptiles, dónde viven, cómo duermen, las partes de su cuerpo y del caparazón, qué diferencia hay entre los machos y las hembras y cómo respiran.

- Sobre el proyecto del agua: qué deportes se pueden practicar en el agua, hay agua en nuestro cuerpo, para qué utilizamos el agua, el agua en el planeta tierra, por qué hay olas en el mar, el ciclo del agua, cómo llega el agua a nuestras casas, las 
tres formas del agua: líquida, sólida y vapor y que no tiene sabor, color, ni olor.

Como limitaciones del estudio presentado, cabe destacar que solamente se ha analizado la dimensión $C$ de la guía CEIN y sería interesante abarcar también la dimensión $\mathrm{A}$ y $\mathrm{B}$ para reflexionar en torno a cuestiones relacionadas con cómo somos y cómo nos organizamos, e involucrar así a todo el centro escolar. Es importante reflexionar sobre estos aspectos para avanzar hacia una escuela intercultural e inclusiva donde todos puedan aprender y participar. Como futuras líneas de investigación, se podría seguir indagando sobre las percepciones de los implicados en la práctica educativa -alumnado, familias, miembros de la comunidad y la docente- a partir de entrevistas en profundidad.

A modo de cierre, cabe decir que en este trabajo se muestra una buena experiencia en la que con la participación conjunta del alumnado, la familia y los voluntarios se genera una educación de calidad, gracias a una docente innovadora que abre su aula permitiendo a las familias y la comunidad en general a que sean partícipes en la educación integral de sus alumnos.

\section{Referencias bibliográficas}

ARNálz, P. (2004): «La educación inclusiva: dilemas y desafíos». Educación, Desarrollo y Diversidad, 7 (2), p. 25-40

BEAUdoIn, N. (2013): Una escuela para cada estudiante. La relación interpersonal, clave del proceso educativo, Narcea, Madrid.

COHEN, E. G. (1999): Organizzare i gruppi cooperativi. Ruoli, funzioni, attività, Erickson, Trento.

Gómez MonTES, J. M. (2005): "Pautas y estrategias para entender y atender la diversidad en el aula», Pulso, 28.

Gregory, G. H. y C. Chapman (2007): Differentiated Instructional Strategies: One Size Doesn't Fit All, Corwin Press, Thousand Oaks, CA.

López Melero, M. (2004): Construyendo una escuela sin exclusiones: una forma de trabajar en el aula con proyectos de investigación, Ediciones Aljibe, Málaga.

MoriñA, A. (2008): La escuela de la diversidad, Síntesis, Madrid.

Pujolìs, P. (2003): Aprendre junts alumnes diferents. Equips d'aprenentatge cooperatiu a l'aula, Eumo Eidtorial, Vic.

PujolÀs, P. (2008): 9 ideas clave: El aprendizaje cooperativo, Graó, Barcelona.

Sales Ciges, A.; R. Ferrández Berrueco, O. Moliner García (2012): «Escuela intercultural inclusiva: estudio de caso sobre procesos de autoevaluación», Revista de Educación, 358, p. 153-173. 
Sales, A., O. Moliner y J. Traver (2010): La construcción de la escuela intercultural inclusiva desde procesos de investigación-acción. Servicio de Publicaciones de la Universitat Jaume I, Castelló.

SÁnCHEZ PALOMINO, A. Y J. CARIÓN (2002): «Una aproximación a la investigación en Educación Especial», Revista de Educación, núm. 327, p. 225-247.

StainBack y Stainback (1999): Aulas inclusivas, Narcea, Madrid.

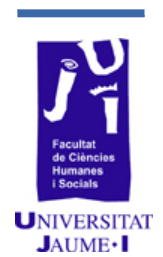

Vieira Parra y PUigdellivol (2013): "¿Voluntarios dentro del aula?» REXE. Revista de Estudios y Experiencias en Educación, 12 (24), p. 37-55.

WALKER, R. (1983): "La realización de estudios de casos en educación. Ética, teoría y procedimientos», Dockrell W. B. y D. HAMILTON, Nuevas reflexiones sobre la investigación educativa, Narcea, Madrid, p. 42-82.

Woods, P. (1987): La escuela por dentro. La etnografía en la investigación educativa, Paidós, Barcelona. 